\title{
Risposte esatte ai Test di Verifica
}

Alluvione di Messina 2009: NGAL in due pazienti con Crush Syndrome

V. Donato, A. Noto, A. Lacquaniti, A. Versaci, M. Giardina, D. Bolignano, F. Spinelli, A. David, M. Buemi

Test di Verifica - 1, Pag. 4

Domanda n. 1: risposta esatta: B

Domanda n. 2: risposta esatta: E

Domanda n. 3: risposta esatta: $F$

Domanda n. 4: risposta esatta: A

Domanda n. 5: risposta esatta: $F$

Domanda n. 6: risposta esatta: D

Sindrome nefrosica steroido-resistente trattata con ACTH

P. Lorusso, I. Cipollini, A. Bottai, G. Barsotti

Test di Verifica - 2, Pag. 7

Domanda n. 1: risposta esatta: D

Domanda n. 2: risposta esatta: B

Domanda n. 3: risposta esatta: D

Un tunnel "da riaprire" dopo tanti anni di dialisi

T. Lusenti, M. Corradini, PL. Macchioni, F. Fiorini

Test di Verifica - 3, Pag. 12

Domanda n. 1: risposta esatta D:

Domanda n. 2: risposta esatta D:

Domanda n. 3: risposta esatta C: 


\section{L'abito non fa il monaco}

\section{S. Michelassi, S. Nigrelli, M. Amidone, S. Lo Caputo, G. Tinacci}

Test di Verifica - 4, Pag. 15

\section{Domanda 1: Glomerulonefrite membrano-proliferativa (GNMP) tipo 1}

La presenza di proliferazione cellulare ed espansione della matrice mesangiale con aspetti "a doppio contorno" di alcune anse capillari per infiltrazione di matrice, la tendenza all'aspetto "lobulare" del glomerulo e la presenza di complessi immuni prevalentemente a livello mesangiale e subendoteliale sono caratteristiche della GNMP tipo 1. La diagnosi di GNMP tipo 1 è compatibile anche col quadro clinico: microematuria, proteinuria, ipertensione, edemi, riduzione funzionale renale, bassi livelli sierici di $\mathrm{C} 3$ con livelli di $\mathrm{C} 4$ ai limiti inferiori del range di normalità (nella GNMP tipo 1 l'attivazione del complemento avviene per la via classica, determinandone una riduzione più o meno rilevante anche a carico delle frazioni precoci). La GM è caratterizzata alla MO solo da ispessimento diffuso delle pareti capillari senza aspetti di proliferazione cellulare o espansione mesangiale significative e senza deposizione di complessi immuni in sede mesangiale; clinicamente il quadro è dominato dalla proteinuria con o senza sindrome nefrosica e non c'è ipocomplementemia. La glomerulonefrite acuta postinfettiva è caratterizzata istologicamente da ipercellularità intraglomerulare dovuta tuttavia a proliferazione endocapillare ed infiltrazione di granulociti neutrofili (e non a proliferazione delle cellule mesangiali) ed i glomeruli sono spesso talmente ipercellulari da riempire quasi tutto lo spazio capsulare e da rendere invisibili anche i lumi capillari; i complessi immuni più caratteristici (humps) sono localizzati a livello subepiteliale; il quadro clinico è solitamente nefritico ed i livelli sierici di C3 sono bassi ma quelli di C4 sono classicamente normali.

\section{Domanda 2: Le forme secondarie}

La GNMP tipo 1 (come gli altri tipi di GNMP) è in generale un tipo non comune di glomerulonefrite ma in particolare la forma idiopatica è molto rara e deve essere una diagnosticata solo per esclusione. Le forme secondarie sono solitamente dovute a malattie da immunocomplessi, più comunemente ad infezione da $\mathrm{HCV}$ o $\mathrm{HBV}$, a crioglobulinemia mista essenziale (non associata ad HCV), a lupus erithematosus systemicus (LES) e a certe infezioni batteriche $o$ parassitarie (ascessi viscerali, endocardite batterica subacuta, infezione di shunt ventricolo-atriale, malaria, schistosomiasi, ecc) ma sono state descritte in numerose altre condizioni (linfoma, leucemia linfatica cronica, deficit congenito di complemento ecc.) (1).

Test di Verifica - 5, Pag. 16

\section{Domanda 3: Numerosi tipi di lesioni glomerulari, tubulo-interstiziali e dei piccoli vasi}

La gamma di nefropatie associate ad HIV è ampia. I primi lavori, pubblicati oltre vent'anni fa, descrissero in pazienti affetti da AIDS una forma di glomerulosclerosi focale segmentale con caratteristica tendenza al collasso della matassa glomerulare che prese il nome di "HIV-associated nephropathy" (HIVAN) $(2,3)$. Oggi sappiamo che in un numero significativo di soggetti affetti da HIV sono documentabili lesioni istologiche "non-HIVAN" come amiloidosi, glomerulonefrite (GN) a lesioni minime, GN a depositi di IgA, GN "lupus-like", GN membranosa, GN membrano-proliferativa, GN postinfettiva, porpora trombotica trombocitopenica, nefrite interstiziale (spesso associata a nefrocalcinosi), ostruzione intratubulare da cristalli, necrosi tubulare acuta. In alcuni di questi casi la patologia renale è attribuita allo stesso HIV, in altri casi a farmaci o a infezioni opportunistiche (4). La presenza di HIVAN correla strettamente con la razza nera. Su 77 biopsie renali ottenute da pazienti HIV positivi, HIVAN fu diagnosticata nel $43 \%$ dei pazienti neri e nello $0 \%$ di pazienti non neri (5). Inoltre HIVAN è generalmente associata a più rapida progressione rispetto alle lesioni non-HIVAN (intervallo di tempo minore fra la biopsia renale e lingresso in dialisi) (6). E probabilmente proprio per questi motivi, cioè perché le prime casistiche pubblicate erano provenienti dagli USA (dove la razza nera è particolarmente frequente) e perché biopsie renali erano verosimilmente eseguite solo nei casi più aggressivi, che la prima lesione ad essere documentata in altissima percentuale in pazienti HIV positivi fu proprio l'HIVAN. 